\title{
On Hamilton-Jacobi Theory as a Classical Root of Quantum Theory
}

\author{
J. Butterfield ${ }^{1}$ \\ All Souls College \\ Oxford OX1 4AL
}

27 February 2003

\begin{abstract}
This paper gives a technically elementary treatment of some aspects of HamiltonJacobi theory, especially in relation to the calculus of variations. The second half of the paper describes the application to geometric optics, the optico-mechanical analogy and the transition to quantum mechanics. Finally, I report recent work of Holland providing a Hamiltonian formulation of the pilot-wave theory.
\end{abstract}

Forthcoming in Elitzur, A.C., Dolev, S., \& Kolenda, N. (editors), Quo Vadis Quantum Mechanics? Possible Developments in Quantum Theory in the 21st Century, New York: Springer, 2004. (The Frontier Series: Monographs and Books on Frontiers of Modern Physics)

'Dont worry, young man: in mathematics, none of us really understands any idea-we just get used to them.'

John von Neumann, after explaining (no doubt very quickly!) the method of characteristics (i.e. Hamilton-Jacobi theory) to a young physicist, as a way to solve his problem; to which the physicist had replied 'Thank you very much; but I'm afraid I still don't understand this method.'

\footnotetext{
${ }^{1}$ email: jb56@cus.cam.ac.uk; jeremy.butterfield@all-souls.oxford.ac.uk
} 


\section{Introduction}

In the eighty years since its discovery in the mid-1920s, quantum mechanics has gone from strength to strength. It has repeatedly been proved successful, to a high degree of accuracy, in domains of application very different from its original one. For example, although it was devised for systems of atomic dimensions $\left(10^{-8} \mathrm{~cm}\right)$, it has since proven accurate for scales much smaller (cf. the nuclear radius of ca. $10^{-12} \mathrm{~cm}$ ) and vastly larger (cf. superconductivity and superfluidity, involving scales up to $10^{-1} \mathrm{~cm}$ ). Similarly, if we think of domains of application, not as length (or energy) scales, but as types of "stuff" to which the theory applies. Though quantum mechanics was first devised to apply to matter (i.e. electrons and protons, the more "obvious" constituents of atoms), it was soon extended to fields, i.e. the electromagnetic field: indeed, matter soon became regarded as excitations in associated fields. Similarly, if we think of domains of application as types of force: though first devised for electromagnetic forces, quantum mechanics now successfully describes the weak and strong forces. Indeed, similarly for 'domains' understood naively, as regions of the universe: quantum mechanics has also been applied with great success to astronomy-the obvious examples being the use of nuclear physics in theories of stellar structure and evolution, and of particle physics in theories of the early universe.

So quantum mechanics has been an amazing success story. I stress this point at the outset, for two reasons. First: it is, unfortunately, all too easy to get used to success. Nowadays, both physicists, for whom the various quantum theories have become everyday professional tools, and the wider scientifically literate public, can easily lose their sense of wonder at this immense success. So it is worth remembering how contingent, and surprising, it is.

My second reason is more specific to work in the foundations and-or philosophy of quantum theory. This work focusses on the interpretative problems, especially the measurement problem, that still confront quantum mechanics, despite its immense empirical success: hence this volume's question 'Quo vadis, quantum mechanics?' Of course, I endorse that focus: it is crucially important to address these problems. But in addressing them, it is salutary to recall this success, as an intellectual backdrop. Indeed, not only is it salutary: it might also be heuristically useful - though of course, different researchers, with their different intellectual temperaments, will take this success to give different heuristic clues about 'Quo vadis, quantum mechanics?'. For example, an Everettian philosopher such as Saunders (??this volume) may see the success of the established quantum theoretic formalisms as supporting their position: certainly, heterodox quantum theories such as dynamical models of wave-function collapse face an enormous task in recovering that success. On the other hand, a theoretical physicist who is searching for a successor to quantum mechanics - whether to solve these interpretative problems or to reconcile the quantum with general relativity's treatment of gravitation, or both (such as 't Hooft, ??this volume) - may scrutinize the details of this empirical success for clues about how present-day quantum mechanics might be an effective, i.e. phenomenological, theory. As 't Hooft wittily puts it: we can ask, 
not 'Quo vadis, quantum mechanics?', but rather 'Unde venis?'-'Where do you come from?'

This paper will likewise ask 'Unde venis, quantum mechanics?': though I humbly admit that I will interpret this question in a retrospective and expository sense, rather than in 't Hooft's wonderfully forward-looking and creative sense. To be specific: I propose to discuss Hamilton-Jacobi theory as a classical root of quantum mechanics.

One part of this story is well known to physicists and philosophers and historians of physics. Namely: Hamilton-Jacobi theory as a method of integrating Hamilton's equations (using Jacobi's theorem, action-angle variables etc.), and the use made of this integration theory in nineteenth century celestial mechanics, and thereby in the old quantum theory.

There is however another part of this story that seems much less known by this community: viz. Hamilton-Jacobi theory understood from the perspective of the calculus of variations (as worked out by such masters as Hilbert and Carathéodory), and how this understanding motivates deBroglie's and Schrödinger's proposal to extend Hamilton's optico-mechanical analogy, thus creating quantum mechanics (as wave mechanics). So I propose to present this part of the story: or rather, since this part could fill a book - selected pieces of it! (My (2003, 2003a) discuss some other, philosophical, aspects.) At the end of the paper, I shall also briefly return to 'Quo vadis?', i.e. to a current interest in the foundations of quantum theory: viz. the pilot-wave theory-on which Hamilton-Jacobi theory casts some light. But I begin, in the next Subsection, with a more detailed prospectus.

\subsection{Introducing Hamilton-Jacobi theory}

Hamilton-Jacobi theory is a general theory, rich in analytic and geometric ideas, that unifies three apparently disparate topics: systems of first order ordinary differential equations, first order partial differential equations, and the calculus of variations. Roughly speaking, Hamilton-Jacobi theory shows that the following problems are equivalent:-

(ODE): solving a canonical system of first order ordinary differential equations $(2 n$ equations for $2 n$ functions of a parameter $t$ in which all variables' first derivatives are given by partial derivatives of one and the same function); e.g. Hamilton's equations in Hamiltonian mechanics.

(PDE): solving a first order partial differential equation in which the unknown function does not occur explicitly; e.g. the Hamilton-Jacobi equation in mechanics.

$(\mathrm{CV})$ : solving the "basic" calculus of variations problem of finding $n$ functions $q_{1}, \ldots, q_{n}$ of a parameter $t$ that make stationary a line-integral of the form $\int L\left(q_{i}, \dot{q}_{i}, t\right) d t$, where the dot denotes differentiation with respect to $t$; e.g. Hamilton's principle in Lagrangian mechanics, or Fermat's principle in geometric optics.

A bit more precisely: elementary Lagrangian and Hamiltonian mechanics show (ODE) and $(\mathrm{CV})$ to be equivalent for the case of fixed end-points. Hamilton-Jacobi 
theory extends this equivalence by considering, not a single solution of the canonical equations (a single line-integral) but a whole field of solutions, i.e. line-integrals along all the curves of a space-filling congruence (so that the end-points lie on hypersurfaces transverse to the congruence). The initial conditions of a problem then become the specification of a function's values on such a hypersurface, instead of an initial configuration and momentum (or an initial and final configuration): hence the occurrence of partial differential equations.

The main aim of this paper is to explain (in an elementary way) these equivalences and some related results. This explanation will later (Sections 7 and 8) provide us with a perspective on the optico-mechanical analogy and quantum mechanics (specifically, wave mechanics). But there is also a pedagogic rationale for presenting these results. Most physicists learn Hamilton-Jacobi theory only as part of analytical mechanics; and almost all the mechanics textbooks present, in addition to the equivalence of $(\mathrm{ODE})$ and $(\mathrm{CV})$ for fixed end-points, only the use of Hamilton-Jacobi theory as a method of integrating Hamilton's equations - indeed rendering the integration trivial. The central result here is Jacobi's theorem: that given a complete integral of the Hamilton-Jacobi equation (typically found by separation of variables), one can obtain solutions of Hamilton's equations just by differentiation. This is a remarkable result, which lies at the centre of a beautiful geometric theory of the integration of first order partial differential equations: a theory which reduces the integration problem to that of integrating a suitable system of ordinary differential equations (the characteristic equations). But almost all the mechanics textbooks present Jacobi's theorem using just canonical transformation theory: as a result, they do not describe this general integration theory - and more generally, they do not show the role of geometric ideas, nor of the calculus of variations with variable end-points.

This textbook tradition is of course understandable. Textbooks must emphasise problem-solving; and the use of a complete integral of the Hamilton-Jacobi equation to solve Hamilton's equations, is crucially important, for several reasons. As to problemsolving, it is 'the most powerful method known for exact integration, and many problems which were solved by Jacobi cannot be solved by other means' (Arnold 1989, p. 261). Besides, it is conceptually important: it leads on to action-angle variables, which are central both to classical mechanics (e.g. in the Liouville-Arnold theorem, and in perturbation theory) and the old quantum theory.

But though understandable, this tradition is also regrettable. For the result is that most physicists understand well only the equivalence of (ODE) and (CV) for fixed end-points, and a part of the equivalence of (PDE) and (ODE) - the part expressed by Jacobi's theorem. Besides, they understand these matters only in the context of mechanics. This is a pity, for two reasons.

First, it is worth stressing that all these equivalences and related other results, are purely mathematical and so entirely general. Second, the equivalences and results that get omitted from most mechanics textbooks are at least as rich as those included; in particular, in their use of geometric ideas. I might add: 'in their use of optical 
ideas'. Indeed, Hamilton developed his work in mechanics in deliberate analogy with his previous work in optics. ${ }^{2}$ And as we shall see: both Fermat's principle (roughly, that a light ray travels the path that takes least time) and Huygens's principle (roughly, that given a wave-front, a later wave-front is the envelope of spherical waves spreading from the points of the given wave-front) stand at the centre of Hamilton-Jacobi theory. They involve each of the above mathematical problems, in optical guise: viz. the description of light in terms of rays (exemplifying (ODE)), in terms of wavefronts (cf. $(\mathrm{PDE})$ ), and by means of variational principles (cf. (CV)).

Accordingly, I propose to expound some of these equivalences and connections, as mathematics (Sections 2 to 6 ). Then I will illustrate them with geometric optics and the optico-mechanical analogy (Sections 7 and 8).

To be both brief and elementary, this exposition must be very selective. In particular, I will say nothing about: (i) weak solutions; (ii) the use of phase space; (iii) issues about the global existence of solutions, including focussing and caustics. ${ }^{3}$ Another omitted topic lies closer to our concerns: I will not present the theory surrounding Jacobi's theorem, i.e. Hamilton-Jacobi theory as an integration theory for first order partial differential equations. For though I have complained that this is absent from the mechanics books, it is in some books on mathematical methods. ${ }^{4}$

Instead, I will adopt an approach that emphasises the calculus of variations. The main ideas here seem to be due to Carathéodory and Hilbert. Here again, I must be selective: I will simply pick out within this approach, one line of thought, found for example in the first half of Rund (1966). (Rund proves some results which I will only state; and he cites the original papers.) Though selective, this exposition will give a good sense of the triangle of equivalences between (ODE), (PDE) and (CV); indeed, we will get such a sense already by the end of Section 3. Sections 4 to 6 will add to this a discussion of three topics, each leading to the next. They are, respectively: Hilbert's independent integral; treating the integration variable of the variational problem on the same footing as the other coordinates; and integration theory.

Thereafter, Sections 7 et seq. return us to physics. Section 7 discusses geometric

\footnotetext{
${ }^{2}$ For a glimpse of the history, which I will not discuss, cf. e.g.: for mechanics, Dugas (1988), Whittaker (1959); for optics, Whittaker (1952), Buchwald (1989); and for mathematics: Kline (1970, Chap. 30).

${ }^{3}$ A few pedagogic references: for (i) Logan (1994, Chap. 3), Stakgold (1967); for (ii), Arnold (1989, Chap.s 8, 9), Littlejohn (1992), Taylor (1996, Section 1.15); for (iii), Arnold (1989, Appendices 11, 16), Benton (1977), Taylor (1996, Section 6.7). Of these topics, (ii) and (iii) are closest to this paper's interests in geometry, and in the transition between classical and quantum mechanics. For (ii), i.e. Hamilton-Jacobi theory in phase space, beautifully illustrates symplectic geometry; and (ii) and (iii) are crucial in both quantization theory and semiclassical mechanics.

${ }^{4}$ Especially Courant and Hilbert (1962, Chap. II.1-8); cf. also e.g. Webster (1950, Chap 2) and John (1971, Chap 1). In order to be elementary, I will also avoid all use of modern differential geometry, including even the distinction between contravariant and covariant indices. Though modern geometry has transformed our understanding of differential equations and the calculus of variations (and the sciences of mechanics and optics), I shall only need the intuitive geometry familiar from multivariable calculus.
} 
optics; and Section 8, the optico-mechanical analogy and wave mechanics. Section 8 also leads us back to the foundations of quantum mechanics: which I take up briefly in (the last) Section 9. Here I will call attention to the role of Hamilton-Jacobi theory in the pilot-wave theory of deBroglie and Bohm; and more specifically advertise Holland's recent work $(2001,2001 \mathrm{a})$, which provides a Hamiltonian formulation of the pilot-wave theory.

\section{From the calculus of variations to the Hamilton- Jacobi equation}

\subsection{The calculus of variations reviewed}

We begin by briefly reviewing the simplest problem of the calculus of variations; with which we will be concerned throughout the paper. This is the variational problem (in a notation suggestive of mechanics)

$$
\delta I:=\delta I\left[q_{i}\right]=\delta \int_{t_{0}}^{t_{1}} L\left(q_{i}, \dot{q}_{i}, t\right) d t=0, \quad i=1, \ldots, n
$$

where [ ] indicates that $I$ is a functional, the dot denotes differentiation with respect to $t$, and $L$ is to be a $C^{2}$ (twice continuously differentiable) function in all $2 n+1$ arguments. $L$ is the Lagrangian or fundamental function; and $\int L d t$ is the fundamental integral. We will discuss this only locally; i.e. we will consider a fixed simply connected region $G$ of a $(n+1)$-dimensional real space $\mathbb{R}^{n+1}$, on which there are coordinates $\left(q_{1}, \ldots, q_{n}, t\right)=:\left(q_{i}, t\right)=:(q, t)$.

The singling out of a coordinate $t$ (called the parameter of the problem), to give a parametric representation of curves $q(t):=q_{i}(t)$, is partly a matter of notational clarity. But it is of course suggestive of the application to mechanics, where $t$ is time, $q$ represents the system's configuration and $\left(q_{i}, t\right)$-space is often called 'extended configuration space' or 'event space'. Besides, the singling out of $t$ reflects the fact that though it is usual to assume that $L$ (and so the fundamental integral) is invariant under arbitrary transformations (with non-vanishing Jacobian) of the $q_{i}$, we do not require the fundamental integral to be independent of the choice of $t$. Indeed we shall see (at the end of this Subsection and in Section 5) that allowing this dependence is necessary for making Legendre transformations. ${ }^{5}$

A necessary condition for $I$ to be stationary at the $C^{2}$ curve $q(t):=q_{i}(t)$-i.e. for $\delta I=0$ in comparison with other $C^{2}$ curves that (i) share with $q(t)$ the endpoints $q\left(t_{0}\right), q\left(t_{1}\right)$ and (ii) are close to $q(t)$ in both value and derivative throughout

\footnotetext{
${ }^{5}$ Of course, the calculus of variations, and Hamilton-Jacobi theory, can be developed on the assumption that the fundamental integral is to be parameter-independent - if it could not be, so much the worse for relativistic theories! But the details, in particular of how to set up a canonical formalism, are different from what follows. For these details, cf. e.g. Rund (1966, Chapter 3).
} 
$t_{0}<t<t_{1}$-is that: $q(t)$ satisfies for $t_{0}<t<t_{1}$ the $n$ second-order Euler-Lagrange (also known as: Euler, or as Lagrange!) equations

$$
\frac{d}{d t} L_{\dot{q}_{i}}-L_{q_{i}}=0 \quad i=1, \ldots, n .
$$

A curve satisfying these equations is called an extremal.

We will not need to linger on the usual derivation of these equations: we will later see them derived without using a single fixed pair of end-points. Nor need we linger on several related matters taken up in the calculus of variations, such as: the distinction between stationarity and extrema (i.e. maxima or minima), in particular the conditions for a curve to be an extremum not just a stationary point (e.g. conditions concerning the second variation of the fundamental integral, or Weierstrass' excess function); the distinction between weak and strong stationary points and extrema; and the use of weaker assumptions about the smoothness of the solution and comparison curves.

But it is important to consider the canonical form of our variational problem. In physics, the most frequent example of this is the expression of Hamilton's principle within Hamiltonian mechanics; i.e. Hamilton's principle with the integrand a function of both $q \mathrm{~s}$ and $p \mathrm{~s}$, which are to be varied independently. But the correspondence between the Lagrangian form of the variational problem (above) and the canonical form is general (purely mathematical).

Thus, under certain conditions the variational problem eq. 2.1 has an equivalent form, whose Euler-Lagrange equations are $2 n$ first order equations. To this end, we introduce "momenta"

$$
p_{i}:=L_{\dot{q}_{i}}
$$

and (recalling that $L$ is $C^{2}$ ) we assume that the Hessian with respect to the $\dot{q}$ s does not vanish in the domain $G$ considered, i.e. the determinant

$$
\left|L_{\dot{q}_{i} \dot{q}_{j}}\right| \neq 0
$$

so that eq. 2.3 can be solved for the $\dot{q}_{i}$ as functions of $q_{i}, p_{i}, t$.

Then the equations

$$
p_{i}=L_{\dot{q}_{i}} \quad \dot{q}_{i}=H_{p_{i}} \quad L\left(q_{i}, \dot{q}_{i}, t\right)+H\left(q_{i}, p_{i}, t\right)=\Sigma_{i} \dot{q}_{i} p_{i}
$$

represent a Legendre transformation and its inverse; where in the third equation $\dot{q}_{i}$ are understood as functions of $q_{i}, p_{i}, t$ according to the inversion of eq. 2.3. The function $H\left(q_{i}, p_{i}, t\right)$ is called the Legendre (or: Hamiltonian) function of the variational problem, and the $q \mathrm{~s}$ and $p \mathrm{~s}$ are called canonically conjugate. It follows that $H$ is $C^{2}$ in all its arguments, $H_{t}=-L_{t}$, and $\left|L_{\dot{q}_{i} \dot{q}_{j}}\right|=\left|H_{p_{i} p_{j}}\right|^{-1}$. Besides, any $H\left(q_{i}, p_{i}, t\right)$ that is $C^{2}$ in all its arguments, and has a non-vanishing Hessian with respect to the $p \mathrm{~s},\left|H_{p_{i} p_{j}}\right| \neq 0$, is the Legendre function of a $C^{2}$ Lagrangian $L$ given in terms of $H$ by eq. 2.5.

Applying this Legendre transformation, the Euler-Lagrange equations eq. 2.2 go over to the canonical system

$$
\dot{q}_{i}=H_{p_{i}} \quad \dot{p}_{i}=-H_{q_{i}}\left(=L_{q_{i}}\right) .
$$


(A curve satisfying these equations is also called an extremal.) These are the EulerLagrange equations of a variational problem equivalent to the original one, in which both $q$ s and $p$ s are varied independently, namely the problem

$$
\delta \int\left(\Sigma_{i} \dot{q}_{i} p_{i}-H\left(q_{i}, p_{i}, t\right)\right) d t=0
$$

(For more details about eq. 2.3 to 2.7, cf. e.g. Arnold (1989, Chap. 3.14, 9.45.C), Courant and Hilbert (1953, Chap. IV.9.3; 1962, Chap. I.6) and Lanczos (1986, Chap. VI.1-4).)

The requirement of a non-vanishing Hessian, eq. 2.4 (equivalently: $\left|H_{p_{i} p_{j}}\right| \neq 0$ ), is a crucial assumption. Note in particular these two consequences.

1) The Hamiltonian cannot vanish identically. Proof: If we differentiate $H=$ $\Sigma \dot{q}_{i} p_{i}-L=0$ with respect to $\dot{q}_{i}$, we get $\Sigma_{i} L_{\dot{q}_{i} \dot{q}_{j}} \dot{q}_{i}=0$; which contradicts eq. 2.4.

2) $L$ cannot be homogeneous of the first degree in the $\dot{q}_{i}$. That is, we cannot have: $L\left(q_{i}, \lambda \dot{q}_{i}, t\right)=\lambda L\left(q_{i}, \dot{q}_{i}, t\right)$. We shall see in Section 5 that this means the fundamental integral cannot be parameter-independent.

\subsection{Hypersurfaces and congruences}

We consider a family of hypersurfaces in our region $G$ of $\mathbb{R}^{n+1}$

$$
S\left(q_{i}, t\right)=\sigma
$$

with $\sigma \in \mathbb{R}$ the parameter labelling the family, and $S$ a $C^{2}$ function (in all $n+1$ arguments). We assume this family covers the region $G$ simply, in the sense that through each point of $G$ there passes a unique hypersurface in the family.

Let $C$ be a curve

$$
q_{i}=q_{i}(t)
$$

of class $C^{2}$, that lies in $G$ and intersects each hypersurface in the family eq. 2.8 just once, but is nowhere tangent to a hypersurface. Then $\sigma$ is a function of $t$ along $C$, with

$$
\Delta:=\frac{d \sigma}{d t}=\Sigma_{i} \frac{\partial S}{\partial q_{i}} \dot{q}_{i}+\frac{\partial S}{\partial t}
$$

By construction $\Delta \neq 0$. We will assume that the Lagrangian $L$ does not vanish along $C$. By a suitable labelling of the family of surfaces, we can secure

$$
\Delta>0 \text { or }<0 \text { according as } L>0 \text { or }<0
$$

for the line-element $\left(q_{i}, \dot{q}_{i}, t\right)$ of $C$. Then a tangential displacement along $C$ from $P:=$ $\left(q_{i}, t\right)$ to $Q:=\left(q_{i}+d q_{i}, t+d t\right)$, i.e. a displacement with components $\left(d q_{i}, d t\right)=\left(\dot{q}_{i}, 1\right) d t$, induces an increment $d \sigma$ in $\sigma$, and an increment $d I=L\left(q_{i}, \dot{q}_{i}, t\right) d t$ in $I=\int L d t$. 
To connect this family of hypersurfaces with the calculus of variations, we now seek values of $\dot{q}_{i}$ at $P$ such that the direction at $P$ of the curve $C,\left(\dot{q}_{i}, 1\right) d t$, makes $d I / d \sigma$ a minimum with $d \sigma$ fixed. A necessary condition is that

$$
\frac{\partial}{\partial \dot{q}_{i}}\left(\frac{d I}{d \sigma}\right)=0, \quad i=1, \ldots, n
$$

But $\frac{d I}{d \sigma}=\frac{L}{\Delta}$ and $\Delta \neq 0$, so that eq. 2.12 reads

$$
\frac{\partial L}{\partial \dot{q}_{i}}-\frac{L}{\Delta} \frac{\partial \Delta}{\partial \dot{q}_{i}}=0
$$

that is, using $\frac{\partial \Delta}{\partial \dot{q}_{i}}=\frac{\partial S}{\partial q_{i}}$ from eq. 2.10,

$$
\frac{\partial L}{\partial \dot{q}_{i}}=\frac{L}{\Delta} \frac{\partial S}{\partial q_{i}}
$$

A curve $C$, or its tangent vector $\left(\dot{q}_{i}, 1\right)$, that satisfies eq. 2.14 , is said to be in the direction of the geodesic gradient determined by the family of surfaces 2.8 .

As it stands, this condition eq. 2.14 can at best yield minima of $d I / d \sigma$; while we are interested in minima of $d I / d t$. But there is a further condition on the family of surfaces eq. 2.8 that implies that curves obeying eq. 2.14 are solutions of the variational problem; or rather, to be precise, extremals.

This condition has two equivalent forms; the first geometric in spirit, the second analytic. They are:

(a): that the quantity $L / \Delta$ is constant on each surface, i.e. there is some real function $\phi$ such that

$$
\frac{L}{\Delta}=\phi(\sigma)
$$

where we are to take the directional arguments in $L$ to refer to the geodesic gradient.

(b): that $S$ solves the Hamilton-Jacobi equation.

It is straightforward to show that (a) implies that we can re-parametrize the family of surfaces in such a way that $L=\Delta$ throughout the region $G$. That is to say: given (a), the family can be re-parametrized so that function $\phi$ is the constant function 1: $\phi(\sigma)=1$. (Proof: any monotonic function $\psi$ gives a re-parametrization of the family, $\psi(S)=\psi(\sigma)$, with $\bar{\Delta}$ defined on analogy with $\Delta$ by $\bar{\Delta}:=\frac{d}{d t} \psi(\sigma)=\psi^{\prime}(\sigma) \Delta$. Choosing $\psi(\sigma):=\int_{\sigma_{0}}^{\sigma} \phi(s) d s\left(\sigma_{0}\right.$ some constant) yields $\psi^{\prime}(\sigma)=\phi(\sigma)$ so that $\frac{L}{\Delta} \equiv \frac{L}{\psi^{\prime}(\sigma) \Delta} \equiv$ $\frac{\phi(\sigma)}{\psi^{\prime}(\sigma)}=1$.)

So to show (a) and (b) equivalent, we will show that:

(i) given (a) in this special form, i.e. given $L=\Delta, S$ solves the Hamilton-Jacobi equation; and conversely

(ii) $S$ solving the Hamilton-Jacobi equation implies that $L=\Delta$.

But it will be clearest, before proving this equivalence, to present two consequences of $L=\Delta$, and introduce some terminology. 
First: $L=\Delta$ implies that the geodesic gradient, eq. 2.14, is now given by

$$
\frac{\partial L}{\partial \dot{q}_{i}}=p_{i}=\frac{\partial S}{\partial q_{i}} \text {. }
$$

where the first equation uses eq. 2.3. Recall now our assumption that the determinant $\left|L_{\dot{q}_{i} \dot{q}_{j}}\right| \neq 0$, so that eq. 2.3 can be solved in $G$ for the $\dot{q}_{i}$ as functions of $q_{i}, p_{i}, t$ : $\dot{q}_{i}=q_{i}\left(q_{i}, p_{i}, t\right)$. This now reads as

$$
\dot{q}_{i}=\dot{q}_{i}\left(q_{i}, \frac{\partial S}{\partial q_{i}}, t\right)
$$

where the right-hand side is a function of $\left(q_{i}, t\right)$ alone (since $S$ is) and has continuous first order derivatives. Then the elementary existence theorem for solutions of first order ordinary differential equations implies that eq. 2.17 defines an $n$-parameter family of curves in the region $G$, such that each point in $G$ has a unique curve pass through it, and each curve is a solution of eq. 2.17 in the sense that the components of its tangent vectors obey eq. 2.17. This family of curves is called the congruence $K$ belonging to the family of surfaces eq. 2.8.

Second: $L=\Delta$ implies that the increment $d I$ in the fundamental integral $I=$ $\int L d t$, in passing from a point $P_{1}$ on the surface $S\left(q_{i}, t\right)=\sigma_{1}$, to an adjacent surface $S=\sigma_{1}+d \sigma$, along a curve of the congruence belonging to the family, obeys

$$
d I=\Delta d t=d \sigma .
$$

Integrating this result along members of the congruence, we get: the integral along a curve of the congruence, from any point $P_{1}$ on the surface $S\left(q_{i}, t\right)=\sigma_{1}$ to that point $P_{2}$ on the surface $S\left(q_{i}, t\right)=\sigma_{2}$ that lies on the same curve of the congruence, is the same for whatever point $P_{1}$ we choose. That is:

$$
\int_{P_{1}}^{P_{2}} L d t=\sigma_{2}-\sigma_{1}
$$

Clearly, the converse also holds: if the fundamental integral taken along curves of the congruence has the same value for two hypersurfaces, however we choose the end-points $P_{1}, P_{2}$ lying in the hypersurfaces, then $L=\Delta$. So a family of surfaces satisfying the condition that $L=\Delta$ is called geodesically equidistant with respect to the Lagrangian L. (Courant and Hilbert (1962, Chap. II.9.2) say 'geodetic', not 'geodesic'; which has the advantage of avoiding "geodesic"s possibly confusing connotations of metric and-or connection.)

Carathéodory called a family of geodesically equidistant hypersurfaces, together with the congruence belonging to it, the complete figure (of the variational problem). As we shall see, the name is apt, since the complete figure is central to Hamilton-Jacobi theory. Also, the congruence is called transversal to the surfaces of the family. The analytical expression of transversality is that for a displacement $\left(\delta q_{i}, \delta t\right)$ tangential to a hypersurface in the family, $\delta S=0$. That is:

$$
\frac{\partial S}{\partial q_{i}} \delta q_{i}+\frac{\partial S}{\partial t} \delta t=0
$$


We turn to showing that: (i) $L=\Delta$ implies that $S$ solves the Hamilton-Jacobi equation.

Proof: Eq. 2.10 yields

$$
L\left(q_{i}, \dot{q}_{i}, t\right)=\Delta:=\frac{d \sigma}{d t}=\Sigma_{i} \frac{\partial S}{\partial q_{i}} \dot{q}_{i}+\frac{\partial S}{\partial t}
$$

where $\dot{q}_{i}$ refers to the direction of the geodesic gradient, eq. 2.17 , i.e. $\dot{q}_{i}=\dot{q}_{i}\left(q_{i}, \frac{\partial S}{\partial q_{i}}, t\right)$. This yields

$$
-\frac{\partial S}{\partial t}=-L\left(q_{i}, \dot{q}_{i}\left(q_{i}, \frac{\partial S}{\partial q_{i}}, t\right), t\right)+\Sigma_{i} \frac{\partial S}{\partial q_{i}} \dot{q}_{i}\left(q_{i}, \frac{\partial S}{\partial q_{i}}, t\right) .
$$

But eq. 2.5 implies that the right-hand side is the Hamiltonian function, but with $p_{i}$ replaced by $\frac{\partial S}{\partial q_{i}}$ in accordance with eq. 2.16. Thus we have

$$
\frac{\partial S}{\partial t}+H\left(q_{i}, \frac{\partial S}{\partial q_{i}}, t\right)=0
$$

which is the Hamilton-Jacobi equation.

This equation is also a sufficient condition of a family of surfaces being geodesically equidistant. That is, (ii): $S$ being a $C^{2}$ solution in $G$ of the Hamilton-Jacobi equation implies that $L=\Delta$, i.e. that the hypersurfaces of constant $S$ are geodesically equidistant.

Proof: Given such a solution $S\left(q_{i}, t\right)$, let us define an assignment to each point of $G$ (sometimes called a field) by

$$
p_{i} \equiv p_{i}\left(q_{i}, t\right):=\frac{\partial S}{\partial q_{i}} .
$$

By eq. 2.4, this determines a field $\dot{q}_{i}$ as in eq. 2.17, and hence a congruence. Then for the given solution $S$, a given member $C$ of the congruence, and two given parameter values $\sigma_{1}, \sigma_{2}$, we form the fundamental integral along $C$ between the points $P_{1}$ and $P_{2}$ where $C$ intersects the hypersurfaces corresponding to the parameter values $\sigma_{1}, \sigma_{2}$. Using the Legendre transformation, eq. 2.5 and the fact that $S$ solves the HamiltonJacobi equation, eq. 2.23, we obtain:

$\int_{P_{1}}^{P_{2}} L d t=-\int_{P_{1}}^{P_{2}}\left[H\left(q_{i}, \frac{\partial S}{\partial q_{i}}, t\right)-\Sigma_{i} p_{i} \dot{q}_{i}\right] d t=\int_{P_{1}}^{P_{2}}\left[\frac{\partial S}{\partial t} d t+\Sigma_{i} \frac{\partial S}{\partial q_{i}} d q_{i}\right]=\int_{P_{1}}^{P_{2}} d S=\sigma_{2}-\sigma_{1}$.

To sum up: a family of hypersurfaces $S=\sigma$ is geodesically equidistant with respect to the Lagrangian $L$ iff $S$ is a solution of the Hamilton-Jacobi equation whose Hamiltonian $H$ corresponds by the Legendre transformation to $L$. And if this holds, the transversality condition, eq. 2.20, can be written (using eq. 2.23 and 2.24) as

$$
p_{i} \delta q_{i}-H\left(q_{i}, p_{i}, t\right) \delta t=0 .{ }^{6}
$$

\footnotetext{
${ }^{6}$ Transversality can also be defined, without any use of a family of hypersurfaces, or even a function $S$, in terms of the fundamental integral being stationary as an end-point of the integral varies on a given single surface. Cf. e.g. Courant and Hilbert (1953, Chap. IV.5.2).
} 


\section{Canonical and Euler-Lagrange equations; fields of extremals}

We now study the properties of a congruence $K$ belonging to a family of geodesically equidistant surfaces. We first show that any curve of such a congruence obeys the canonical and Euler-Lagrange equations. Then we develop the ideas of: a field $q_{i}, p_{i}$ in the region $G$; and a field belonging to a family of (not necessarily geodesically equidistant) hypersurfaces. Finally we characterize those fields belonging to geodesically equidistant hypersurfaces.

\subsection{Canonical and Euler-Lagrange equations}

The family eq. 2.8 defines an assignment of $p_{i}:=\frac{\partial S}{\partial q_{i}}$ to each point of a member $C$ of the congruence $K$. If we differentiate the definition of $p$ i.e. eq. 2.24 with respect to $t$ along $C$, and we differentiate the Hamilton-Jacobi equation eq. 2.23, and we then use the fact (from eq. 2.6) that $\dot{q}_{i}=\frac{\partial H}{\partial p_{i}}$, we can eliminate the second derivatives of $S$ that arise in the differentiations, and get:

$$
\dot{p}_{i}=-\frac{\partial H}{\partial q_{i}} .
$$

To this, we can adjoin $\dot{q}_{i}=\frac{\partial H}{\partial p_{i}}$, so as to get $2 n$ first order ordinary differential equations obeyed by members of $K$

$$
\dot{p}_{i}=-\frac{\partial H}{\partial q_{i}} \quad ; \quad \dot{q}_{i}=\frac{\partial H}{\partial p_{i}} .
$$

Note that according to this deduction, these two groups of equations have different statuses, despite their symmetric appearance. $\dot{p}_{i}=-\frac{\partial H}{\partial q_{i}}$ depends on $K$ belonging to a family of geodesically equidistant surfaces (i.e. on the Hamilton-Jacobi equation ). But $\dot{q}_{i}=\frac{\partial H}{\partial p_{i}}$ are identities derived from the theory of the Legendre transformation (cf. eq. 2.6). But this difference is not peculiar to our deduction's use of hypersurfaces. The same difference occurs in derivations of these equations in the calculus of variations with fixed end-points: in the most familiar case, in Lagrangian mechanics i.e. without use of the canonical integral; (cf. e.g. Lanczos (1986, p. 166-7).

From the canonical equations we can deduce the (Lagrangian form of the) EulerLagrange equations. We substitute $p_{i}=\frac{\partial L}{\partial \dot{q}_{i}}$ in the left-hand side, and $\frac{\partial H}{\partial q_{i}}=-\frac{\partial L}{\partial q_{i}}$ in the right-hand side, of the first of eq. 3.2, to get

$$
\frac{d}{d t} \frac{\partial L}{\partial \dot{q}_{i}}-\frac{\partial L}{\partial q_{i}}=0
$$




\section{$3.2 \quad$ Fields}

To discuss fields, we need first to consider parametric representations of an arbitrary smooth congruence of curves covering our region $G$ simply. That is, we consider a congruence represented by $n$ equations giving $q_{i}$ as $C^{2}$ functions of $n$ parameters and $t$

$$
q_{i}=q_{i}\left(u_{\alpha}, t\right) \quad i=1, \ldots, n
$$

where each set of $n u_{\alpha}=\left(u_{1}, \ldots, u_{n}\right)$ labels a unique curve in the congruence. Thus there is a one-to-one correspondence $\left(q_{i}, t\right) \leftrightarrow\left(u_{\alpha}, t\right)$ in appropriate domains of the variables, with non-vanishing Jacobian

$$
\left|\frac{\partial q_{i}}{\partial u_{\alpha}}\right| \neq 0
$$

Such a congruence determines tangent vectors $\left(\dot{q}_{i}, 1\right)$ at each $\left(q_{i}, t\right)$; and thereby also values of the Lagrangian $L\left(q_{i}\left(u_{\alpha}, t\right), \dot{q}_{i}\left(u_{\alpha}, t\right), t\right)$ and of the momentum

$$
p_{i}=p_{i}\left(u_{\alpha}, t\right)=\frac{\partial L}{\partial \dot{q}_{i}} .
$$

Conversely, a set of $2 n C^{2}$ functions $q_{i}, p_{i}$ of $\left(u_{\alpha}, t\right)$ as in eqs 3.4 and 3.6, with the $q$ s and $p$ s related by $p_{i}=\frac{\partial L}{\partial \dot{q}_{i}}$, determines a set of tangent vectors, and so a congruence. Such a set of $2 n$ functions is called a field; and if all the curves of the congruence are extremals (i.e. solutions of the Euler-Lagrange equations), it is called a field of extremals.

We say a field belongs to a (not necessarily geodesically equidistant) family of hypersurfaces given by eq. 2.8 iff throughout the region $G$ eq.s 2.16 and 3.4 are together satisfied, i.e. iff we have

$$
p_{i}=\frac{\partial}{\partial q_{i}} S\left(q_{i}, t\right)=\frac{\partial}{\partial q_{i}} S\left(q_{i}\left(u_{\alpha}, t\right), t\right) .
$$

One can show that a field belongs to a family of hypersurfaces iff for all indices $\alpha, \beta=$ $1, \ldots, n$, the Lagrange brackets of the parameters of the field, i.e.

$$
\left[u_{\alpha}, u_{\beta}\right]:=\Sigma_{i}\left(\frac{\partial q_{i}}{\partial u_{\alpha}} \frac{\partial p_{i}}{\partial u_{\beta}}-\frac{\partial q_{i}}{\partial u_{\beta}} \frac{\partial p_{i}}{\partial u_{\alpha}}\right)
$$

vanish identically. ${ }^{7}$

We say that a field $q_{i}=q_{i}\left(u_{\alpha}, t\right), p_{i}=p_{i}\left(u_{\alpha}, t\right)$ is canonical if the $q_{i}, p_{i}$ satisfy eq. 3.2. Now we will show that if a canonical field belongs to a family of hypersurfaces eq. 2.8 , then the members of the family are geodesically equidistant.

\footnotetext{
${ }^{7}$ Cf. Rund (1966, p. 28-30). Warning: the role of Lagrange brackets in this theory is sometimes omitted even in excellent accounts, e.g. Courant and Hilbert (1962, Chap. II.9.4).
} 
Proof: Differentiating eq. 3.7 with respect to $t$ along a member of the congruence, and substituting on the left-hand side from the first of eq. 3.2, we get

$$
-\frac{\partial H}{\partial q_{i}}=\frac{\partial^{2} S}{\partial q_{i} \partial q_{j}} \dot{q}_{j}+\frac{\partial^{2} S}{\partial q_{i} \partial t}
$$

By the second of eq. 3.2, this is

$$
\frac{\partial^{2} S}{\partial q_{i} \partial t}+\frac{\partial H}{\partial q_{i}}+\frac{\partial H}{\partial p_{j}} \frac{\partial^{2} S}{\partial q_{i} \partial q_{j}}=0
$$

which is

$$
\frac{\partial}{\partial q_{i}}\left(\frac{\partial S}{\partial t}+H\left(q_{j}, \frac{\partial S}{\partial q_{j}}, t\right)\right)=0
$$

which is integrated immediately to give

$$
\frac{\partial S}{\partial t}+H\left(q_{j}, \frac{\partial S}{\partial q_{j}}, t\right)=f(t)
$$

with $f$ an arbitrary function of $t$ only. Now we argue (in the usual way, for the calculus of variations) that this function can be absorbed in $H$. For suppose the given Lagrangian were replaced by $\tilde{L}=L+f(t)$. The path-independence of the integral $\int f(t) d t$ implies that $L$ and $\tilde{L}$ give equivalent variational problems, i.e. the same curves give stationary values for both $\int L d t$ and $\int \tilde{L} d t$. Besides, the definition of $p_{i}$, eq. 2.3, and the canonical equations eq. 2.6 are unaffected, the only change in our formalism being that $H$ is replaced by $\tilde{H}=H-f(t)$. So assuming that $L$ is replaced by $\tilde{L}$ means that eq. 3.12 reduces to the Hamilton-Jacobi equation, eq. 2.23. The result now follows from result (ii) at the end of Section 2.2.

This result is a kind of converse of our deduction of eq. 3.2. We can sum up this situation by saying that the canonical equations characterize any field belonging to a family of geodesically equidistant hypersurfaces.

Finally, we should note an alternative to our order of exposition. We assumed at the outset a family of hypersurfaces, and then discussed an associated congruence and field. But one can instead begin with a single arbitrary surface; then define the notion of an extremal being transverse to the surface (in terms of the fundamental integral being stationary as an end-point varies on the surface - cf. footnote 6 ); then define a field of such transverse extremals; and finally define other surfaces, geodesically equidistant to the given one, as surfaces $S=$ constant, where $S\left(q_{i}, t\right)$ is defined to be the value of the fundamental integral taken along a transverse extremal from the given surface $(S=0)$ to the point $\left(q_{i}, t\right)$. This alternative order of exposition is adopted by Courant and Hilbert (1962, Chap. II.9.2-5), and (more briefly) by Born and Wolf (1999, Appendix I.2-4). It has the mild advantage over ours of clearly displaying the choice of an arbitrary initial surface; (which accords with the solution of a partial differential equation involving an arbitrary function just as the solution of an ordinary differential equation involves an arbitrary constant or constants). It will also come up again in Sections 6 and 7. 


\section{Hilbert's independent integral}

A canonical field belonging to a geodesically equidistant family of hypersurfaces defines a line-integral which is independent of its path of integration. This integral, named after its discoverer Hilbert, is important not only in Hamilton-Jacobi theory, but also in aspects of the calculus of variations which we do not discuss, e.g. the study of conditions for the fundamental integral to take extreme values.

Suppose we are given a geodesically equidistant family of hypersurfaces covering region $G$ simply. Consider two arbitrary points $P_{1}, P_{2} \in G$ lying on hypersurfaces $S=\sigma_{1}, S=\sigma_{2}$ respectively; and consider an arbitrary $C^{1}$ curve $C: q_{i}=q_{i}(t)$ lying in $G$ and joining $P_{1}$ and $P_{2}$. We will write the components of the tangent vector $\left(d q_{i} / d t, 1\right)$ of $C$ as $\left(q_{i}^{\prime}, 1\right)$; for we continue to use the dot ${ }^{*}$ for differentiation along the geodesic gradient of the field belonging to $S$. Now consider the integral along $C$ of $d S$, so that the integral is trivially path-independent:

$$
J:=\int_{P_{1}}^{P_{2}} d S\left(q_{i}, t\right)=\sigma_{2}-\sigma_{1}=\int_{P_{1}}^{P_{2}}\left(\frac{\partial S}{\partial q_{i}} q_{i}^{\prime}+\frac{\partial S}{\partial t}\right) d t
$$

We can apply $p_{i}=\frac{\partial S}{\partial q_{i}}$ and the Hamilton-Jacobi equation to the first and second terms of the integrand respectively, to get a path-independent integral

$$
J=\int_{P_{1}}^{P_{2}}\left(p_{i} q_{i}^{\prime}-H\left(q_{i}, p_{i}, t\right)\right) d t=\sigma_{2}-\sigma_{1}
$$

We can also Legendre transform to eliminate the $p_{i}$ in favour of $\dot{q}_{i}$, getting

$$
J=\int_{P_{1}}^{P_{2}}\left(L\left(q_{i}, \dot{q}_{i}, t\right)+\frac{\partial L}{\partial \dot{q}_{i}}\left(q_{i}^{\prime}-\dot{q}_{i}\right)\right) d t=\sigma_{2}-\sigma_{1} .
$$

It is in this form that $J$ is usually called the Hilbert integral.

A field

$$
q_{i}=q_{i}\left(u_{\alpha}, t\right) \quad p_{i}=p_{i}\left(u_{\alpha}, t\right)
$$

(assumed to belong to a family of hypersurfaces in the sense of eq. 3.7) is called a Mayer field if substituting $q_{i}, p_{i}$ in the integral in eq. 4.2 yields an integral that is path-independent. So we have seen that a canonical field is a Mayer field. One can show that the converse holds, i.e. any Mayer field is canonical (Rund 1966, p. 33). So we have the result: a Mayer field is a canonical field belonging to a family of geodesically equidistant hypersurfaces. (It can also be shown that every extremal curve can be imbedded in a Mayer field.)

Combining this with the results of Section 3, we also have: the field eq. 4.4 is a Mayer field iff the Lagrange brackets $\left[u_{\alpha}, u_{\beta}\right]$ vanish and the field obeys the canonical equations eq. 3.2. 


\section{The parameter as an additional $q$-coordinate}

As we said at the start of Section 2.1, our theory has depended from the outset on the choice of $t$; (cf. the fundamental integral eq. 2.1). Indeed, we saw at the end of Section 2.1 that the non-vanishing Hessian eq. 2.4 implies that $L$ cannot be homogeneous of the first degree in the $\dot{q}_{i}$; i.e. we cannot have for all $\lambda \in \mathbb{R}, L\left(q_{i}, \lambda \dot{q}_{i}, t\right)=\lambda L\left(q_{i}, \dot{q}_{i}, t\right)$. And we shall shortly see that this implies that the fundamental integral cannot be parameter-independent.

But for some aspects of the theory, especially the next Section's discussion of Hamilton-Jacobi theory as an integration theory for first order partial differential equations, it is both possible and useful to treat $t$ as a coordinate on a par with the $q$ s. So in this Section, we describe such a treatment and the gain in symmetry it secures.

To have some consistency with our previous notation, we first consider a Lagrangian $L\left(q_{\alpha}, \dot{q}_{\alpha}, t\right)$ with $n-1$ coordinates $q_{\alpha}$, a parameter $t$ and derivatives $\dot{q}_{\alpha}=d q_{\alpha} / d t$. So note: in this Section, Greek indices run from 1 to $n-1$. So the fundamental integral along a curve $C: q_{\alpha}=q_{\alpha}(t)$ in a suitable region $G$ of $\mathbb{R}^{n}$ joining points $P_{1}, P_{2}$ with parameters $t_{1}, t_{2}$ is

$$
I=\int_{t_{1}}^{t_{2}} L\left(q_{\alpha}, \dot{q}_{\alpha}, t\right) d t
$$

Now we introduce a real $C^{1}$ function $\tau(t)$ which is such that $d \tau / d t>0$ for all values of $t$ under consideration, but is otherwise arbitrary. We write derivatives with respect to $\tau$ using dashes, so that

$$
q_{\alpha}^{\prime}=\dot{q}_{\alpha}\left(\frac{d t}{d \tau}\right)
$$

So with $\tau_{1}:=\tau\left(t_{1}\right), \tau_{2}:=\tau\left(t_{2}\right)$, we can write eq. 5.1 as

$$
I=\int_{\tau_{1}}^{\tau_{2}} L\left(q_{\alpha}, q_{\alpha}^{\prime} \frac{d \tau}{d t}, t\right) \frac{d t}{d \tau} d \tau
$$

If we now write $q_{n}$ for $t$, so that we can write the coordinates on $\mathbb{R}^{n}$ as

$$
q_{i}=\left(q_{\alpha}, t\right)=\left(q_{\alpha}, q_{n}\right) i=1, \ldots, n \text { and write } \frac{d t}{d \tau}=q_{n}^{\prime} \neq 0
$$

then we can write eq. 5.3 as

$$
I=\int_{\tau_{1}}^{\tau_{2}} L^{*}\left(q_{i}, q_{i}^{\prime}\right) d \tau
$$

where we have defined

$$
L^{*}\left(q_{i}, q_{i}^{\prime}\right):=L^{*}\left(q_{\alpha}, t, q_{\alpha}^{\prime}, q_{n}^{\prime}\right):=L\left(q_{\alpha}, \frac{q_{\alpha}^{\prime}}{q_{n}^{\prime}}, t\right) .
$$

We stress that the values of the integrals eq.s 5.1 and 5.5 are equal. But the latter is by construction parameter-independent, since the choice of $\tau$ is essentially 
arbitrary. Also $L^{*}$ is by construction positively homogeneous of the first degree in the $q_{i}^{\prime}=\left(q_{\alpha}^{\prime}, q_{n}^{\prime}\right)$-i.e. for all positive numbers $\lambda, L^{*}\left(q_{i}, \lambda q_{i}^{\prime}\right)=\lambda L\left(q_{i}, q_{i}^{\prime}\right)$-irrespective of the form of the given Lagrangian $L$. In fact one can easily show that these two features are equivalent.

For the purposes of the next Section, we will now express the canonical equations of our variational problem, eq.s 5.1 or 5.5, in the new notation. But note: the total differentiation on the left-hand sides of the canonical equations will still be differentiation with respect to the original parameter $t$ - and so indicated by a dot.

Writing the conjugate momenta of $L^{*}$ as $p^{*}$ for the moment, we have

$$
p_{\alpha}^{*}=\frac{\partial L^{*}}{\partial q_{\alpha}^{\prime}}=\frac{\partial L}{\partial \dot{q}_{\alpha}} \frac{1}{q_{n}^{\prime}} q_{n}^{\prime}=p_{\alpha}
$$

so that these are identical with the original conjugate momenta; and so we will drop the ${ }^{*}$ in $p_{\alpha}^{*}$. So the canonical equations for the indices $1, \ldots, n-1$ are given, with the original Hamiltonian (Legendre) function $H\left(q_{\alpha}, p_{\alpha}, t\right)$ as defined in eq. 2.5, by

$$
\dot{q}_{\alpha}=\frac{\partial H}{\partial p_{\alpha}}, \quad \dot{p}_{\alpha}=-\frac{\partial H}{\partial q_{\alpha}} .
$$

On the other hand, for the new $p_{n}$, we have

$$
p_{n}:=\frac{\partial L^{*}}{\partial q_{n}^{\prime}}=L-\Sigma_{\alpha} \frac{\partial L}{\partial \dot{q}_{\alpha}} \frac{q_{\alpha}^{\prime}}{q_{n}^{\prime}}=L-\Sigma_{\alpha} p_{\alpha} \dot{q}_{\alpha} .
$$

Comparing with the definition eq. 2.5 of the Hamiltonian (Legendre) function, this is

$$
p_{n}+H\left(q_{\alpha}, p_{\alpha}, t\right)=0 .
$$

So differentiating $p_{n}$ with respect to the original parameter $t$ along an extremal gives

$$
\dot{p}_{n}:=\frac{d p}{d t}=-\frac{d H}{d t}=-\frac{\partial H}{\partial t}=-\frac{\partial H}{\partial q_{n}}
$$

which fits well with eq. 5.8; (here $-\frac{d H}{d t}=-\frac{\partial H}{\partial t}$ follows as usual from the canonical equations, i.e. from $H$ 's Poisson bracket with itself vanishing identically). But note that we also have $\dot{q}_{n}:=\frac{d t}{d t}=1 \neq \frac{\partial H}{\partial p_{n}}=-1$.

However, we can use the Hamilton-Jacobi equation to overcome this last "wrinkle". i.e. to get a greater degree of symmetry. We can write the Hamilton-Jacobi equation of our variational problem eq. 5.1 as

$$
\Phi\left(q_{i}, \frac{\partial S}{\partial q_{i}}\right)=H\left(q_{\alpha}, \frac{\partial S}{\partial q_{\alpha}}, q_{n}\right)+\frac{\partial S}{\partial q_{n}}=0
$$

where $\Phi$ is defined as a function of $2 n$ variables by

$$
\Phi\left(q_{i}, p_{i}\right):=H\left(q_{\alpha}, p_{\alpha}, q_{n}\right)+p_{n} .
$$


Now if the $p_{\alpha}$ in eq. 5.13 refer to a field of extremals belonging to a solution $S\left(q_{\alpha}, q_{n}\right)$ of the Hamilton-Jacobi equation, so that $p_{\alpha}=\frac{\partial S}{\partial q_{\alpha}}$, then by eq.s 5.10 and 5.12 , we also have: $p_{n}=\frac{\partial S}{\partial q_{n}}$. Besides, eq. 5.13 implies immediately

$$
\frac{\partial \Phi}{\partial q_{i}}=\frac{\partial H}{\partial q_{i}} \quad, \quad \frac{\partial \Phi}{\partial p_{\alpha}}=\frac{\partial H}{\partial p_{\alpha}} \quad, \quad \frac{\partial \Phi}{\partial p_{n}}=1\left(=\dot{q}_{n} \equiv \frac{d t}{d t}\right) .
$$

It follows that we can write the canonical equations eq. 5.8, together with the relations for $q_{n}, p_{n}$, in a completely symmetrical way in terms of $\Phi$ as

$$
\dot{q}_{i}=\frac{\partial \Phi}{\partial p_{i}} \quad, \quad \dot{p}_{i}=-\frac{\partial \Phi}{\partial q_{i}}
$$

where, note again, the dot denotes differentiation with respect to $t$.

\section{Integrating first order partial differential equa- tions}

As mentioned in Section 1, we will not expound the usual approach (with Jacobi's theorem) to Hamilton-Jacobi theory as an integration theory for first order partial differential equations. ${ }^{8}$ Instead, we will in this Section briefly introduce another approach which exploits the results and concepts of the previous Sections; (for more details, cf. Rund, 1966, Chap. 2.8).

We will consider a partial differential equation of the form

$$
\Phi\left(q_{i}, \frac{\partial S}{\partial q_{i}}\right)=0, i=1, \ldots, n ; \quad \text { with } \quad \frac{\partial \Phi}{\partial p_{i}} \neq 0 \text { for at least one } i
$$

and $\Phi$ of class $C^{2}$ in all $2 n$ arguments. One of the $i$ for which $\frac{\partial \Phi}{\partial p_{i}} \neq 0$ may be identified with $t$, but this is not necessary: as in the previous Section, our discussion can treat all coordinates of $\mathbb{R}^{n}$ on an equal footing. We shall also assume that (as suggested by the Hamilton-Jacobi equation) the unknown function $S$ does not occur explicitly in the equation; but this is not really a restriction, since one can show that the general case, i.e. an equation in which $S$ occurs, can be reduced to the form of eq. 6.1 by introducing an additional independent variable.

So the initial value problem we are to solve is: to find a function $S\left(q_{i}\right)\left(q_{i} \in G\right)$ that satisfies eq. 6.1 and that assumes prescribed values on a given $(n-1)$-dimensional $C^{2}$ surface, $V$ say, in $G$. We will indicate how to explicitly construct such a function by using a congruence of "canonical" curves which solve a canonical system of ordinary differential equations; (so we reduce the integration of the partial differential equation

\footnotetext{
${ }^{8}$ For an exposition cf. the references in footnote 4 . As to the history: Whittaker $(1959$, p. 264,316$)$ reports that this theory was developed by Pfaff and Cauchy in 1815-1819, using earlier results by Lagrange and Monge; i.e. well before Hamilton's and Jacobi's work!
} 
to the problem of integrating ordinary differential equations). This canonical system of equations will be suggested by our previous discussion; and the strategy of the construction will be to adjust the congruence of curves from an initial rather arbitrary congruence, to one that provides a solution to eq. $6.1 .^{9}$

Thus our previous discussion (especially Sections 3 and 5) suggests we should consider the system of $2 n$ ordinary differential equations, with a new parameter $s$

$$
\dot{q}_{i}:=\frac{d q_{i}}{d s}=\frac{\partial \Phi\left(q_{j}, p_{j}\right)}{\partial p_{i}} \quad, \quad \dot{p}_{i}:=\frac{d p_{i}}{d s}=-\frac{\partial \Phi\left(q_{j}, p_{j}\right)}{\partial q_{i}}
$$

These are called the characteristic equations of eq. 6.1. A curve $q_{i}=q_{i}(s)$ of $\mathbb{R}^{n}$ that satisfies them is called a characteristic curve of eq. 6.1 ; it will be an extremal of a problem in the calculus of variations if eq. 6.1 is the Hamilton-Jacobi equation of such a problem. Our approach to integrating eq. 6.1 applies to these characteristic equations theorems about the existence and uniqueness of solutions of ordinary differential equations, so as to secure the existence and uniqueness of solutions to eq. 6.1.

Let us consider an $(n-1)$-parameter congruence of characteristic curves, with parameters $u_{1}, \ldots, u_{n-1}$, so that we write

$$
q_{i}=q_{i}\left(s, u_{\alpha}\right) \quad, \quad p_{i}=p_{i}\left(s, u_{\alpha}\right) .
$$

Since $\Phi$ is $C^{2}$, it follows from eq. 6.2 that the functions 6.3 are $C^{2}$ in $s$. We will also assume that these functions are $C^{2}$ in the $u_{\alpha}$; and that this congruence covers the region $G$ simply, with

$$
\frac{\partial\left(q_{1}, q_{2}, \ldots, q_{n}\right)}{\partial\left(s, u_{1}, \ldots, u_{n-1}\right)} \neq 0
$$

so that we can invert the first set of eq. 6.3 for $\left(s, u_{\alpha}\right)$, getting

$$
s=s\left(q_{i}\right), u_{\alpha}=u_{\alpha}\left(q_{i}\right) .
$$

We shall also write (in $G$ ):

$$
\phi\left(s, u_{\alpha}\right):=\Phi\left(q_{i}\left(s, u_{\beta}\right), p_{i}\left(s, u_{\beta}\right)\right) .
$$

One can now show:

(i): $\phi$ of eq. 6.6 is an integral of the characteristic equations eq. 6.2 , i.e. $\frac{d \phi}{d s}=0$;

(ii): the Lagrange brackets $\left[u_{\alpha}, s\right]$ and $\left[u_{\alpha}, u_{\beta}\right]$ are constant along any member of the congruence defined by eq. 6.2.

We now make some assumptions about the relation of our characteristic congruence to the given surface $V$. We will assume that through each point of $V$ there passes a unique member of the congruence, and that the congruence is nowhere tangent to $V$.

\footnotetext{
${ }^{9}$ We remark at the outset that since - as in previous Sections-we work in a "configuration space", not its twice-dimensional "phase space", there are many "canonical congruences", rather than a unique one; so that this sort of adjustment is possible.
} 
Thus each point in $V$ is assigned $n-1$ parameter values $u_{\alpha}$ and a value of $s$; so we can write $s$ on $V$ as a $C^{2}$ function of $u_{\alpha}$, the parameters of the unique curve through the point. Let us write this as $s=\sigma\left(u_{\alpha}\right)$, so that the functions $a_{I}\left(u_{\alpha}\right)$ defined by

$$
a_{i}\left(u_{\alpha}\right):=q_{i}\left(\sigma\left(u_{\alpha}\right), u_{\alpha}\right)
$$

are also $C^{2}$. Finally we will suppose that we seek a solution of eq. 6.1 which takes the values $c\left(u_{\alpha}\right)$ on $V, c$ prescribed $C^{2}$ functions.

That completes the assumptions needed for the construction of a (local) solution of eq. 6.1 (and the proof of its uniqueness). We end this Section by briefly describing the first steps of the construction.

The theory of first order ordinary differential equations implies that the congruence of characteristic curves for eq. 6.2 is determined if the values of $q_{i}$ and $p_{i}$ are prescribed on $V$. The initial values of $q_{i}$ are of course to be given by the $a_{i}$ of eq. 6.7. But as to the initial values of the $p_{i}$, i.e. $b_{i}$ defined by

$$
b_{i}\left(u_{\alpha}\right):=p_{i}\left(\sigma\left(u_{\alpha}\right), u_{\alpha}\right),
$$

we have some choice. The strategy of the construction is, roughly speaking, to define a function $S$ on $G$, in such a way that when we adjust the $b_{i}$ so that $p_{i}=\frac{\partial S}{\partial q_{i}}, S$ becomes a solution of eq. 6.1 in $G$, possessing the required properties.

We now define a function $z=z\left(s, u_{\alpha}\right)$ on $G$ in terms of $V$, the values $c\left(u_{\alpha}\right)$ prescribed on $V$ and the given congruence; in effect, this $z$ will be the desired $S$, once the $b_{i}$ are suitably adjusted. For each point $P \in G$, with its $n$ parameter values $\left(s, u_{\alpha}\right)$, the $s$-value of the intersection with $V$ of the unique curve through $P$ is given by $s=\sigma\left(u_{\alpha}\right)$. We define the value of $z$ at $P$ by

$$
z\left(s, u_{\alpha}\right):=c\left(u_{\alpha}\right)+\int_{\tau=\sigma\left(u_{\alpha}\right)}^{s} \Sigma_{i}\left[p_{i}\left(\tau, u_{\alpha}\right) \frac{\partial \Phi\left(q_{i}\left(\tau, u_{\alpha}\right), p_{i}\left(\tau, u_{\alpha}\right)\right)}{\partial p_{i}}\right] d \tau,
$$

where the integration is to be taken along the curve through $P$, from its point of intersection with $V$, to $P$.

We will not go further into the construction of the desired $S$, except to make two remarks. (1): Note that eq. 6.9 implies in particular that $z\left(\sigma\left(u_{\alpha}\right), u_{\alpha}\right)=c\left(u_{\alpha}\right)$.

(2): Differentiating eq. 6.9 with respect to $s$ and using the first set of eq. 6.2 yields

$$
\dot{z} \equiv \frac{\partial z}{\partial s}=\Sigma_{i} p_{i} \frac{\partial \Phi}{\partial p_{i}}=\Sigma_{i} p_{i} \dot{q}_{i}
$$

This is analogous to the relation $\dot{S}=\Sigma_{i} p_{i} \dot{q}_{i}$ between a scalar function, such as a solution $S$ of the Hamilton-Jacobi equation and the field $q_{i}, p_{i}$ belonging to it, i.e. the field such that $p_{i}=\frac{\partial S}{\partial q_{i}}$; cf. eq. 3.7. Indeed, if we use eq. 6.5 to define a function $S$ on $G$ by

$$
S\left(q_{i}\right):=z\left(s\left(q_{i}\right), u_{\alpha}\left(q_{i}\right)\right)
$$


then one can show (again, we omit the details!) that:

(i) we can adjust the $b_{i}$ so as to make $p_{i}=\frac{\partial S}{\partial q_{i}}$ hold; and

(ii) that this adjustment makes $S$, as defined by eq. 6.11 (and so 6.9), a solution of eq. 6.1 with the required properties.

\section{The characteristic function and geometric optics}

In this Section, we follow in Hamilton's $(1833,1834$ !) footsteps. We introduce the Hamilton-Jacobi equation via the characteristic function (as do most mechanics textbooks); and then apply these ideas to geometric optics - so our discussion will (at last!) make contact with physics. The main point will be that the correspondence in our formalism between canonical extremals and geodesically equidistant hypersurfaces underpins the fact that both the corpuscular and wave conceptions of light can account for the phenomena, viz. reflection and refraction, described by geometric optics. ${ }^{10}$

We assume that our region $G \subset \mathbb{R}^{n+1}$ is sufficiently small that between any two points $P_{1}=\left(q_{1 i}, t_{1}\right), P_{2}=\left(q_{2 i}, t_{2}\right)$ there is a unique extremal curve $C$. To avoid double subscripts, we will in this Section sometimes suppress the $i$, writing $P_{1}=\left(q_{1}, t_{1}\right), P_{2}=$ $\left(q_{2}, t_{2}\right)$ etc. Then the value of the fundamental integral along $C$ is a well-defined function of the coordinates of the end-points; which we call the characteristic function and write as

$$
S\left(q_{1}, t_{1} ; q_{2}, t_{2}\right)=\int_{t_{1}}^{t_{2}} L d t=\int_{t_{1}}^{t_{2}}\left(\Sigma_{i} p_{i} \dot{q}_{i}-H\right) d t=\int \Sigma_{i} p_{i} d q_{i}-H d t
$$

where the integral is understood as taken along the unique extremal $C$ between the end-points, and we have used eq. 2.5.

Making arbitrary small displacements $\left(\delta q_{1}, \delta t_{1}\right),\left(\delta q_{2}, \delta t_{2}\right)$ at $P_{1}, P_{2}$ respectively, and using the fact that the integral is taken along an extremal, we get for the variation in $S$

$$
\begin{array}{r}
\delta S:=S\left(q_{1}+\delta q_{1}, t_{1}+\delta t_{1} ; q_{2}+\delta q_{2}, t_{2}+\delta t_{2}\right)-S\left(q_{1}, t_{1} ; q_{2}, t_{2}\right)= \\
\frac{\partial S}{\partial t_{1}} \delta t_{1}+\frac{\partial S}{\partial t_{2}} \delta t_{2}+\Sigma_{i} \frac{\partial S}{\partial q_{1 i}} \delta q_{1 i}+\Sigma_{i} \frac{\partial S}{\partial q_{2 i}} \delta q_{2 i}=\left[\Sigma_{i} p_{i} \delta q_{i}-H\left(q_{j}, p_{j}, t\right) \delta\right]_{t_{1}}^{t_{2}}
\end{array}
$$

Since the displacements are independent, we can identify each of the coefficients on the two sides of the last equation in eq. 7.2 , getting

$$
\begin{array}{cc}
\frac{\partial S}{\partial t_{2}}=-\left[H\left(q_{i}, p_{i}, t\right)\right]_{t=t_{2}} \quad, \quad \frac{\partial S}{\partial q_{2 i}}=\left[p_{i}\right]_{t=t_{2}} \\
\frac{\partial S}{\partial t_{1}}=\left[H\left(q_{i}, p_{i}, t\right)\right]_{t=t_{1}} \quad, \quad \frac{\partial S}{\partial q_{1 i}}=-\left[p_{i}\right]_{t=t_{1}}
\end{array}
$$

\footnotetext{
${ }^{10}$ This is an example of what philosophers call "under-determination of theory by data". The escape from this sort of quandary is of course the consideration of other phenomena: in this case, the nineteenth-century study of diffraction and interference, which led to the rise of wave optics-cf. the start of Section 8.
} 
in which the $p_{i}$ refer to the extremal $C$ at $P_{1}$ and $P_{2}$.

These equations are remarkable, since they enable us, if we know the function $S\left(q_{1}, t_{1}, q_{2}, t_{2}\right)$ to determine all the extremals (in mechanical terms: all the possible motions of the system) — without solving any differential equations! For suppose we are given the initial conditions $\left(q_{1}, p_{1}, t_{1}\right)$, (i.e., in mechanical terms: the configuration and canonical momenta at time $\left.t_{1}\right)$, and also the function $S$. The $n$ equations $\frac{\partial S}{\partial q_{1}}=-p_{1}$ in eq. 7.4 relate the $n+1$ quantities $\left(q_{2}, t_{2}\right)$ to the given constants $q_{1}, p_{1}, t_{1}$. So in principle, we can solve these equations by a purely algebraic process, to get $q_{2}$ as a function of $t_{2}$ and the constants $q_{1}, p_{1}, t_{1}$. Finally, we can get $p_{2}$ from the $n$ equations $p_{2}=\frac{\partial S}{\partial q_{2}}$ in eq. 7.3. So indeed the extremals are found without performing integrations, i.e. just by differentiation and elimination: a very remarkable technique. ${ }^{11}$

Substituting the second set of equations of eq. 7.3 in the first yields

$$
\frac{\partial S}{\partial t_{2}}+H\left(q_{2}, \frac{\partial S}{\partial q_{2}}, t_{2}\right)=0
$$

So the characteristic function $S\left(q_{1}, t_{1} ; q_{2}, t_{2}\right)$ considered as a function of the $n+1$ $\operatorname{arguments}\left(q_{2}, t_{2}\right)=\left(q_{2 i}, t_{2}\right)$ (i.e. with $\left(q_{1}, t_{1}\right)$ fixed) satisfies the Hamilton-Jacobi equation.

Assuming that this solution $S$ is $C^{2}$, it follows from result (ii) of Section 2.2 that $S$ defines a family of geodesically equidistant hypersurfaces, namely the geodesic hyperspheres (for short: geodesic spheres) with centre $P_{1}=\left(q_{1}, t_{1}\right)$. Thus the sphere with radius $R$ is given by the equation

$$
S\left(q_{1}, t_{1} ; q_{2}, t_{2}\right)=R
$$

with $\left(q_{1}, t_{1}\right)$ considered fixed. So every point $P_{2}$ on this sphere is connected to the fixed centre $P_{1}=\left(q_{1}, t_{1}\right)$ by a unique extremal along which the fundamental integral has value $R$. These extremals cut the spheres eq. 7.6 transversally.

These geodesic spheres about the various points $P_{1}$ are special families of hypersurfaces. For by taking envelopes of these spheres, we can build up successive members of an arbitrary family of geodesically equidistant hypersurfaces. This is the basic idea of Huygens' principle in geometric optics. Though Huygens first stated this idea as part of his wave theory of light, it can be stated entirely generally. Indeed, there is a rich theory here. We will not enter details ${ }^{12}$, but just state the main idea.

Thus consider some arbitrary solution $S\left(q_{i}, t\right)$ of the Hamilton-Jacobi equation

$$
\frac{\partial S}{\partial t}+H\left(q_{i}, \frac{\partial S}{\partial q_{i}}, t\right)=0
$$

\footnotetext{
${ }^{11}$ As Hamilton of course realized. He writes, in the impersonal style of the day, that 'Mr Hamilton's function $S \ldots$ must not be confounded with that so beautifully conceived by Lagrange for the more simple and elegant expression of the known differential equations [i.e. L]. Lagrange's function states, Mr Hamilton's function would solve the problem. The one serves to form the differential equations of motion, the other would give their integrals' (1834, p. 514).

${ }^{12}$ For details, cf. Baker and Copson (1950), Herzberger (1958). In optics, the Hamilton-Jacobi equation is often called the eikonal equation.
} 
and thereby the canonical field (congruence) $K$ belonging to it, for which $p_{i}=\frac{\partial S}{\partial q_{i}}$. Let $h_{1}, h_{2}$ be two hypersurfaces corresponding to values $\sigma_{1}, \sigma_{2}$ of $S$, i.e. $\left(q_{i}, t\right) \in h_{j},(j=$ $1,2)$ iff $S\left(q_{i}, t\right)=\sigma_{j}$. Let $P_{1}$ be in $h_{1}$, and let the canonical extremal $C$ through $P_{1}$ intersect $h_{2}$ in $P_{2}$. Then we already know from eq. 2.19 that the fundamental integral along $C$ is

$$
\int_{P_{1}}^{P_{2}} L d t=\sigma_{2}-\sigma_{1}
$$

so that $P_{2}$ is in the geodesic sphere centred on $P_{1}$ with radius $\sigma_{2}-\sigma_{1}$. Huygens' principle states that more is true: $h_{2}$ is the envelope of the set of geodesic spheres of radius $\sigma_{2}-\sigma_{1}$ with centres on the hypersurface $h_{1}$.

As a final task for this Section, we briefly illustrate our formalism with another topic in geometric optics: namely, Fermat's "least time" principle, which states (roughly speaking) that a light ray between spatial points $P_{1}$ and $P_{2}$ travels by the path that makes stationary the time taken. This illustration has two motivations. First: together with the next Section's discussion, it will bring out the optico-mechanical analogy - and so prompt the transition to wave mechanics.

Second: it illustrates how our formalism allows $t$ to be a coordinate like the $q_{i}$, even though it is singled out as the integration variable; (cf. Section 5). In fact, there are subtleties here. For if one expresses Fermat's principle using time as the integration variable, one is led to an integrand that is in general, e.g. for isotropic media, homogeneous of degree 1 in the velocities $\dot{q}_{i}$; and as noted in remark 2) at the end of Section 2.1, this conflicts with our requirement of a non-vanishing Hessian (eq. 2.4), i.e. with our construction of a canonical formalism. So illustrating our formalism with Fermat's principle in fact depends on using a spatial coordinate as integration variable (parameter along the light's path). As we will see in a moment, this gives an integrand which is in general, even for isotropic media, not homogeneous of degree 1 in the velocities - so that we can apply the theory of Sections 2 onwards.

So now our preferred coordinate $t$ will be (not time, as it will be in mechanics) but one of just three spatial coordinates $\left(q_{1}, q_{2}, t\right)$ for ordinary Euclidean space. In fact, applications of geometric optics, e.g. to optical instruments which typically have an axis of symmetry, often suggest a natural choice of the coordinate $t$.

At a point $P=\left(q_{1}, q_{2}, t\right)$ in an optical medium, a direction is given by direction ratios $\left(\dot{q}_{1}, \dot{q}_{2}, \dot{t}\right)=\left(\dot{q}_{1}, \dot{q}_{2}, 1\right)$. (So note: the subscripts 1 and 2 now refer to the first and second of three spatial axes "at a single time" - and not to initial and final configurations.) The speed of a ray of light through $P$ in this direction will in general depend on both position and direction, i.e. on the five variables $\left(q_{i}, \dot{q}_{i}, t\right), i=1,2$; and so the speed is denoted by $v\left(q_{i}, \dot{q}_{i}, t\right)$. If $c$ is the speed of light in vacuo, the refractive index is defined by

$$
n\left(q_{i}, \dot{q}_{i}, t\right):=c / v\left(q_{i}, \dot{q}_{i}, t\right) .
$$

If $n$ is independent of the directional arguments $\dot{q}_{i}$ (respectively: positional arguments $\left.q_{i}, t\right)$, the medium is called isotropic (respectively: homogeneous). 
Now let the curve $C: q_{i}=q_{i}(t)$ represent the path of a light-ray between two points $P_{1}, P_{2}$ with parameter values $t=t_{1}, t=t_{2}$. Then the time taken to traverse this curve (the optical length of the curve) is

$$
T=\int_{t_{1}}^{t_{2}} \frac{d s}{v}=\int_{t_{1}}^{t_{2}} \frac{n\left(q_{i}, \dot{q}_{i}, t\right)}{c}\left[\left(\dot{q}_{1}\right)^{2}+\left(\dot{q}_{2}\right)^{2}+1\right]^{\frac{1}{2}} d t=\int_{t_{1}}^{t_{2}} L d t
$$

where we have defined

$$
L\left(q_{i}, \dot{q}_{i}, t\right):=\frac{n\left(q_{i}, \dot{q}_{i}, t\right)}{c}\left[\left(\dot{q}_{1}\right)^{2}+\left(\dot{q}_{2}\right)^{2}+1\right]^{\frac{1}{2}} .
$$

However, our discussion will not be concerned with this special form of $L$. We will only require that $L$ be $C^{2}$, and that the Hessian does not vanish, i.e. eq. 2.4 holds. One immediately verifies that this is so for isotropic media; (in fact the Hessian is $\left.\frac{n\left(q_{i}, t\right)^{2}}{c^{2}}\left[\left(\dot{q}_{1}\right)^{2}+\left(\dot{q}_{2}\right)^{2}+1\right]^{-2} \neq 0\right)$.

We can now connect our discussion with the principles of Fermat and Huygens. We can again take Fermat's principle in the rough form above, viz. that a light ray between points $P_{1}$ and $P_{2}$ travels by the path that makes stationary the time taken. It follows that if light is instantaneously emitted from a point-source located at $P_{1}=\left(q_{1 i}, t_{1}\right)$ (where now we revert to using ' 1 ' to indicate an initial location), then after a time $T$ the light will register on a surface, $F(T)$ say, such that each point $P_{2}=\left(q_{2 i}, t_{2}\right)$ on $F(T)$ (where similarly, ' 2 ' indicates a final location) is joined to $P_{1}$ by an extremal along which the fundamental integral assumes the common value $T$. This surface is the wave-front for time $T$, due to the point-source emission from $P_{1}$. Clearly, the family of wave-fronts, as $T$ varies, is precisely the family of geodesic spheres (for $L$ as in eq. 7.11) around $P_{1}$.

Using the Hamilton-Jacobi equation eq. 7.7 (now with just three independent variables $\left.q_{1}, q_{2}, t\right)$, we can readily generalize this, so as to describe the construction of successive wave-fronts, given an initial wave-front. Given an arbitrary solution $S\left(q_{1}, q_{2}, t\right)$ of eq. 7.7, and an initial hypersurface $h_{1}$ given by $S\left(q_{i}, t\right)=\sigma_{1}$, we can construct at each point $P_{1} \in h_{1}$ the unique extremal of the canonical field belonging to the family of hypersurfaces of constant $S$. By Fermat's principle, each such extremal can represent a ray emitted from $P_{1}$. If we define along each such extremal the point $P_{2}$ such that that fundamental integral $\int_{P_{1}}^{P_{2}} L d t$ attains the value $T$, then the locus of these points $P_{2}$ is the surface $S=\sigma_{1}+T$. Thus we construct a family of geodesically equidistant hypersurfaces. ${ }^{13}$ To sum up: each solution of the Hamilton-Jacobi equation represents a family of wave-fronts, and the canonical field belonging to a family represents the corresponding light rays.

\footnotetext{
${ }^{13}$ The vector $p_{i}=\frac{\partial S}{\partial q_{i}}$ is longer the more rapidly $S$ increases over space, i.e. the more rapidly the light's time of flight increases over space. So Hamilton called $p_{i}$ the vector of normal slowness.
} 


\section{From the optico-mechanical analogy to wave me- chanics}

The rise of wave optics in the nineteeth century led to geometric optics being regarded as the short-wavelength regime of a wave theory of light. So its equations and principles, such as the Hamilton-Jacobi equation and Fermat's and Huygens' principles, came to be seen as effective statements derived in the short-wavelength limit of the full wave theory. But the details of these derivations are irrelevant here. ${ }^{14}$

For us the relevant point is that (as is often remarked: e.g. Synge (1954, Preface), Rund (1966, p. 100)): once one considers this development, together with the optico-mechanical analogy as stated so far (i.e. as it stood for Hamilton), it is natural to speculate that there might be a wave mechanics, just as there is a wave optics. That is, it is natural to speculate that classical mechanics might describe the shortwavelength regime of a wave mechanics, just as geometric optics describes the shortwavelength regime of a wave optics. This is of course precisely what deBroglie, and then Schrödinger, did. To be more specific, using our Hamilton-Jacobi perspective: they proposed that $S$ represented, not an ensemble of systems each fully described by its classical mechanical state $(q, p)$, but a property of an individual system. ${ }^{15}$

In this Section, we give a simple sketch of this proposal. But we shall not give details of deBroglie's and Schrödinger's own arguments, which are subtle and complicated: (Dugas (1988, Part V, Chap. 4) gives some of this history). Our sketch is formal, though in the textbook tradition (Rund (1966, pp. 99-109) and Goldstein (1950, pp. 307-314)); (various books give fuller accounts e.g. using the concepts of Fourier analysis and the group velocity of a wave-packet, e.g. Messiah (1966, pp. 50-64), Gasiorowicz (1974, pp. 27-32)). More precisely: we will first describe how when we apply HamiltonJacobi theory to a classical mechanical system, the $S$-function defines for each time $t$ surfaces of constant $S$ in configuration space, so that by varying $t$ we can calculate the velocity with which these "wave-fronts" propagate (in configuration space). So far, so classical. But then we will postulate that these wave-fronts are surfaces of constant phase of a time-dependent complex-valued wave-function on configuration space. This will lead us, with some heuristic steps, to the Schrödinger equation and so to wave mechanics.

Let us consider a classical mechanical system with holonomic ideal constraints,

\footnotetext{
${ }^{14}$ cf. e.g. Born and Wolf (1999, Chap. 3.1, 8.3.1); Taylor (1996, Section 6.6-6.7) is a brief but advanced mathematical discussion.

${ }^{15}$ Of course, successful proposals often seem "natural" in hindsight; and some authors (e.g. Goldstein (1950, p. 314)) maintain that deBroglie's and Schrödinger's proposal would have seemed merely idle speculation if it had been made independently of the introduction of Planck's constant and the subsequent struggles of the old quantum theory. Indeed, even in that context it was obviously: (i) daringly imaginative (witness the fact that the $S$ wave propagates in multi-dimensional configuration space); and (ii) confusing (witness the interpretative struggles over the reality of the wave-function). In any case, whether the proposal was natural or not-after all, 'natural' is a vague word —all can now agree that their achievement was enormous.
} 
on which the constraints are solved so as to give a $n$-dimensional configuration space $Q$, on which the $q_{i}$ are independent variables. More technically, $Q$ is a manifold, on which the $q_{i}$ are a coordinate system, and on which the kinetic energy defines a metric. But we shall not go into this aspect: we shall simply assume $Q$ is equipped with the usual Euclidean metric on $\mathbb{R}^{n}$, and that the $q_{i}$ are rectangular coordinates. We further assume that any constraints are time-independent (scleronomous); i.e. any configuration in $Q$ is possible for the system throughout the time period in question. The result of these assumptions is that the region $G \subset \mathbb{R}^{n+1}$ for which the formalism of Sections 2 has been developed is now assumed to be an 'event space' or 'extended configuration space' of the form $Q \times T$, where $T \subset \mathbb{R}$ is some real interval representing a period of time. Finally, we will assume that our system is conservative, with energy $E$.

Now we will presume, without rehearsing the usual equations (cf. especially Section 2.1 and eq.s 7.1 to 7.5 ), that using the above assumptions, the Lagrangian and Hamiltonian mechanics of our system has been set up. So if $S\left(q_{i}, t\right)=\sigma$ is a family of geodesically equidistant hypersurfaces associated with the system (each hypersurface $n$-dimensional), the family covering our region $G$ simply, then $S$ satisfies the HamiltonJacobi equation in the form $\frac{\partial S}{\partial t}+E=0$. This can be immediately integrated to give, for some function $S^{*}$ of $q_{i}$ only,

$$
S\left(q_{i}, t\right)=S^{*}\left(q_{i}\right)-E t .
$$

(So the $p_{i}$ of the canonical field depend only on $S^{*}: p_{i}:=\frac{\partial S}{\partial q_{i}}=\frac{\partial S^{*}}{\partial q_{i}}$.) So the hypersurfaces of our family can be written as

$$
S^{*}\left(q_{i}\right)=E t+\sigma .
$$

For any fixed $t$, a hypersurface of constant $S$, considered as a hypersurface in the configuration space $Q$ (a hypersurface of dimension $n$-1, i.e. co-dimension 1 ), e.g. the surface $S\left(q_{i}, t\right)=\sigma_{1}$, coincides with a hypersurface of constant $S^{*}$ : for this example, the surface $S^{*}=\sigma_{1}+E t$. But while the surfaces of constant $S^{*}$ are time-independent, the surfaces of constant $S$ vary with time. So we can think of the surfaces of constant $S$ as propagating through $Q$. With this picture in mind, let us calculate their velocity.

(We can state the idea of surfaces in $Q$ of constant $S$ more rigorously, using our assumption that the region $G \subset \mathbb{R}^{n+1}$ is of the form $Q \times T$. This implies that any equation of constant time, $t=$ constant, defines a $n$-dimensional submanifold of $G$ which is a "copy" of $Q$; let us call it $Q_{t}$. Each hypersurface in eq. 8.2 defines a $(n-1)$-dimensional submanifold of $Q_{t}$ (a hypersurface in $Q_{t}$ of co-dimension 1 ) given by

$$
S^{*}\left(q_{i}\right)=E t+\text { constant, (with } t=\text { constant). }
$$

Then, as in the previous paragraph: fixing the constant $\sigma$ but letting $t$ vary, and identifying the different copies $Q_{t}$ of $Q$, we get a family of $(n-1)$-dimensional submanifolds of $Q$, parametrized by $t$. This can be regarded as a wave-front propagating over time through the configuration space $Q$.) 
Let us fix a constant $\sigma$ and a time $t$; let $P=\left(q_{i}\right) \in Q$ be a point on the surface $S=S^{*}-E t=\sigma$; and consider the normal to this surface (pointing in the direction of propagation) at $P$. (So the $i$ th component $n_{i}$ of the unit normal is $n_{i}=\left|\nabla S^{*}\right|^{-1} \frac{\partial S^{*}}{\partial q_{i}}$.) Consider a point $P^{\prime}=\left(q_{i}+d q_{i}\right)$ that lies a distance $d s$ from $P$ along this normal: (so $\left.d q_{i}=n_{i} d s\right) . P^{\prime}$ is on a subsequent wave-front (i.e. with the same value $\sigma$ of $S$, but not of $\left.S^{*}\right)$ at time $t+d t$, where by eq. 8.2

$$
d S^{*}=\Sigma_{i} \frac{\partial S^{*}}{\partial q_{i}} d q_{i}=E d t
$$

which, dividing by $d s$, yields

$$
\frac{d S^{*}}{d s} \equiv\left|\nabla S^{*}\right|=\Sigma_{i} \frac{\partial S^{*}}{\partial q_{i}} \frac{d q_{i}}{d s}=E \frac{d t}{d s} .
$$

But we also have

$$
p_{i}=\frac{\partial S^{*}}{\partial q_{i}} \Rightarrow p:=|p|=\left|\nabla S^{*}\right| .
$$

Combining these equations, eq. 8.5 and 8.6, we deduce that the speed $u$ of the wavefront $S=\sigma$, i.e. $u:=\frac{d s}{d t}$, is

$$
u=\frac{E}{p}
$$

So far, so classical. But now we postulate that the wave-fronts eq. 8.2 (or 8.3) are surfaces of constant phase of a suitable time-dependent complex-valued function $\psi$ on $Q$. This postulate, together with some heuristic steps (including a judicious identification of Planck's constant!), will give us a heuristic derivation of the Schrödinger equation. We will assume to begin with that we can write the postulated function $\psi=\psi\left(q_{i}, t\right)$ as

$$
\psi=R\left(q_{i}, t\right) \exp \left[-2 \pi i\left(\nu t-\phi\left(q_{i}\right)\right)\right],
$$

with $R$ and $\phi$ real; so that $\nu t-\phi$ is the phase, and (apart from $R$ 's possible $t$ dependence) $\nu$ is the frequency associated with $\psi$. Then our postulate is that there is some constant $h$ such that

$$
h\left(\nu t-\phi\left(q_{i}\right)\right)=\left(E t-S^{*}\left(q_{i}\right)\right)
$$

But this must hold for all $q_{i}, t$, so that

$$
E=h \nu \quad ; \quad S^{*}\left(q_{i}\right)=h \phi\left(q_{i}\right) .
$$

So the postulated frequency is proportional to the system's energy. Then, using our previous calculation of the speed $u$, and the relation $u=\lambda \nu$ with $\lambda$ the wavelength, we deduce that the wavelength is inversely proportional to the magnitude of the system's momentum. That is:

$$
u=\lambda \nu=\frac{E}{p} \Rightarrow \lambda=\frac{h}{p} .
$$


Substituting eq. 8.10 in eq. 8.8 , we can write $\psi$ as

$$
\psi=R\left(q_{i}, t\right) \exp \left[\frac{2 \pi i}{h}\left(S^{*}\left(q_{i}\right)-E t\right)\right]=R\left(q_{i}, t\right) \exp \left[\frac{i}{\hbar}\left(S^{*}\left(q_{i}\right)-E t\right)\right]
$$

where we have defined $\hbar:=\frac{h}{2 \pi}$.

Assuming now that $R$ has no $q_{i}$-dependence, differentiation of eq. 8.12 with respect to $q_{i}$ yields

$$
\frac{\partial \psi}{\partial q_{i}}=\frac{i}{\hbar} \frac{\partial S^{*}}{\partial q_{i}} \psi
$$

Recalling that $p_{i}=\frac{\partial S^{*}}{\partial q_{i}}$, this is an eigenvalue equation, and suggests that we associate with the $i$ th component of momentum $p_{i}$ of a system whose $R$ has no $q_{i}$-dependence, the operator $\hat{p}_{i}$ on wave-functions $\psi$ defined by

$$
\hat{p}_{i}:=\frac{\hbar}{i} \frac{\partial}{\partial q_{i}}, i=1, \ldots, n .
$$

Let us postulate this association also for $q_{i}$-dependent $R$. Then this suggests we also associate with the energy of the system, the operator $\hat{H}$ on wave-functions defined by

$$
\hat{H}:=H\left(q_{i}, \hat{p}_{i}, t\right),
$$

(where we understand $q_{i}$, and functions of it, as operating on wave-functions by ordinary multiplication).

But assuming now that $R$ has no $t$-dependence, differentiation of eq. 8.12 with respect to $t$ yields

$$
i \hbar \frac{\partial \psi}{\partial t}=E \psi
$$

suggesting we should associate with the energy of a system, the operator $\hat{E}$ on wavefunctions defined by

$$
\hat{E}:=i \hbar \frac{\partial}{\partial t}
$$

(By the way, this definition is also motivated by treating time as a coordinate along with the $q_{i}$; cf. the discussion in Section 5. Thus eq. 8.14 suggests we define $\hat{p}_{n+1}:=\frac{\hbar}{i} \frac{\partial}{\partial t}$; and when this is combined with eq. 8.17 , we get

$$
\hat{p}_{n+1}+\hat{E}=0
$$

which is analogous to eq. 5.10.)

If for general $R\left(q_{i}, t\right)$ we endorse both these suggestions - i.e. we identify the actions on eq. 8.12 of these two suggested operators, eq. 8.15 and 8.17 -then we get

$$
\hat{H} \psi=i \hbar \frac{\partial \psi}{\partial t}
$$

which, once we identify $h$ as Planck's constant, is the Schrödinger equation. 


\section{A glance at the pilot-wave theory}

So much by way of sketching the Hamilton-Jacobi perspective on the heuristic route to wave mechanics. In this final Section, I will briefly return to this volume's question ' $Q u o$ vadis, quantum mechanics?', i.e. to the foundations of quantum theory. First, I want to stress that Hamilton-Jacobi theory remains an important ingredient in various research programmes in this field. Prominent among these is the trajectory representation of quantum mechanics, pioneered by Floyd, and Faraggi and Matone. I cannot go into details, but would recommend, as places to begin reading, both Floyd (2002) and Faraggi and Matone (2000). (Besides, Section 1 of the latter ends with some references to other research programmes that use Hamilton-Jacobi theory.)

I shall instead end on Hamilton-Jacobi theory in the context of another prominent research programme (related to the trajectory representation): deBroglie's and Bohm's pilot-wave theory. Again, this is a large topic, and we only wish to advertise the recent work of Holland (2001, 2001a).

First, we recall (Bohm (1952, p. 169), Bohm and Hiley (1993, p. 28), Holland (1993, pp. 69,134)) that:

(i): Writing $\psi=R\left(q_{i}, t\right) \exp \left(i S\left(q_{i}, t\right) / \hbar\right)(R, S$ real $)$ in the one-particle Schrödinger equation, eq. 8.19 with $\hat{H}:=\frac{\hbar^{2}}{2 m} \nabla^{2}+V$ gives

$$
\frac{\partial S}{\partial t}+\frac{1}{2 m}(\nabla S)^{2}+Q+V=0 \text { with } Q:=\frac{-\hbar^{2}}{2 m} \frac{\nabla^{2} R}{R}
$$

which looks like the classical Hamilton-Jacobi equation (cf. eq. 2.23) of a particle in an external potential that is the sum of $V$ and $Q$, which Bohm called the "quantum potential'; indeed Bohm and Hiley call eq. 9.1 the "quantum Hamilton-Jacobi equation"; and

$$
\frac{\partial \rho}{\partial t}+\frac{1}{m} \nabla \cdot(\rho \nabla S)=0 \text { with } \rho:=R^{2} .
$$

(ii): These equations suggest the quantum system comprises both a wave, propagating according to the Schrödinger equation, and a particle, which has (a) a continuous trajectory governed by the wave according to the guidance equation

$$
m \frac{d q_{i}}{d t}=\left.\frac{\partial S}{\partial q_{i}}\right|_{q_{i}=q_{i}(t)}
$$

and (b) a probability distribution given at all times by $|\psi|^{2}=R^{2}$.

Besides, comments and equations similar to those in (i) and (ii) apply when we insert $\psi=R \exp (i S / \hbar)$ into the many-particle Schrödinger equation (Bohm (1952, p. 174), Bohm and Hiley (1993, p. 56 et seq.), Holland (1993, pp. 277 et seq.)).

So far, so good. But Holland (2001, p. 1044) points out that the relation of pilotwave theory to classical Hamilton-Jacobi theory is not transparent. In particular, he points out:

1): The guidance law eq. 9.3 is 'something of an enigma'. It looks like one half of 
a canonical transformation that trivializes the motion of a classical system (by transforming to a set of phase space coordinates that are constant in time. But what about the other half; and more generally, can eq. 9.3 be somehow related to a Hamiltonian or Hamilton-Jacobi theory?

2): $Q$ 's dependence on $S$ (through eq. 9.2) means that the "quantum HamiltonJacobi equation" eq. 9.1 in effect contains higher derivatives of $S$-wholly unlike a classical Hamilton-Jacobi equation.

So Holland undertakes an extensive investigation of this relation. More precisely, he undertakes to formulate the pilot-wave theory as a Hamiltonian theory. He does this by assessing a treatment of $Q$ as a field function of $q_{i}$ on a par with the classical potential $V$; i.e. a treatment that takes as the Hamiltonian of the (one-particle) system

$$
H\left(q_{i}, p_{i}, t\right)=\frac{1}{2 m} \Sigma_{i} p_{i}^{2}+Q\left(q_{i}, t\right)+V\left(q_{i}, t\right)
$$

He emphasises that such a treatment faces three obstacles. In brief, they are:

(a): As we just mentioned in 2), $Q$ depends on $S$ and so presumably, by $p=\frac{\partial S}{\partial q}$, on $p$. So in a Hamiltonian (phase space) treatment, it seems wrong to take $Q$ as a function only of $q$.

(b): The free choice of initial positions and momenta in a Hamiltonian treatment will mean that most motions, projected on $q$, do not give the orthodox quantal distribution, in the way that eq. 9.3 and $|\psi|^{2}=R^{2}$ does.

(c): Is such a treatment compatible with the Hamiltonian description of the Schrödinger equation? For it to be so, we have to somehow formulate the particle-wave interaction so as to prevent a back-reaction on the wave.

However, Holland goes on to show $(2001,2001 \mathrm{a})$ that these obstacles can be overcome. That is, he vindicates the proposal, eq. 9.4, with a Hamiltonian theory of the interacting wave-particle system. But we cannot enter details. It must suffice to list some features of his work. In short, his approach: ble;

(1): generalizes a canonical treatment of a classical particle and associated ensem-

(2): necessitates the introduction of an additional field of which the particle is the source;

(3): makes the "quantum Hamilton-Jacobi equation" and the continuity equation eq. 9.2 (and other equations for the evolution of particle and field variables) come out as Hamilton equations;

(4): interprets $p=\frac{\partial S}{\partial q}$ as a constraint on the phase space coordinates of the waveparticle system;

(5): gives a general formula expressing the condition that the particle's phase space distribution, projected on $q$, gives the orthodox quantal distribution; and finally,

(6): yields a Hamilton-Jacobi theory of the wave-particle system.

To conclude: I hope to have shown that Hamilton-Jacobi theory, understood from the perspective of the calculus of variations, gives us insight into both mechanics and optics - and that, as illustrated by this last Section, Hamilton-Jacobi theory is an 
important ingredient in current attempts to answer the question, 'Quo vadis, quantum mechanics?'.

Acknowledgements:-

It is a pleasure to thank: Avshalom Elitzur and Nancy Kolenda for a superb meeting; the participants, other audiences, and Alexander Afriat, Robert Bishop, Michael Hall, and especially Ned Floyd and Peter Holland, for conversations and comments on a previous version.

\section{References}

V. Arnold (1989), Mathematical Methods of Classical Mechanics, Springer-Verlag (second edition).

B. Baker and E. Copson (1950), The Mathematical Theory of Huygens' Principle, Oxford University Press.

S. Benton (1977), The Hamilton-Jacobi Equation: a Global Approach, Academic Press.

D. Bohm (1952), 'A suggested interpretation of the quantum theory in terms of "hidden" variables, I', Physical Review 85, pp. 166-180.

D. Bohm and B. Hiley (1993), The Undivided Universe, Routledge.

M. Born and E. Wolf (1999), Principles of Optics, Cambridge University Press (7th edition).

J. Buchwald (1989), The Rise of the Wave Theory of Light, University of Chicago Press.

J. Butterfield (2003), 'David Lewis Meets Hamilton and Jacobi', forthcoming in a supplementary issue of Philosophy of Science.

J. Butterfield (2003a), 'Some Aspects of Modality in Analytical mechanics', forthcoming in Formal Teleology and Causality, ed. M. Stöltzner, P. Weingartner, Paderborn: Mentis.

R. Courant and D. Hilbert (1953), Methods of Mathematical Physics, volume I, WileyInterscience (Wiley Classics 1989).

R. Courant and D. Hilbert (1962), Methods of Mathematical Physics, volume II, WileyInterscience (Wiley Classics 1989).

R. Dugas (1988), A History of Mechanics, Dover; reprint of a 1955 French original.

A. Faraggi and M. Matone (2000), 'The Equivalence Postulate of Quantum Mechanics', International Journal of Modern Physics A15, pp. 1869-2017; and available at hep-th/9809127.

E. Floyd (2002), 'The Philosophy of the Trajectory Representation of Quantum Mechanics', in Gravitation and Cosmology: From the Hubble Radius to the Planck Scale, Proceedings of a Symposium in Honor of J.P.Vigier's 80th Birthday, Kluwer; and available at quant-ph/0009070.

S. Gasiorowicz (1974), Quantum Physics, John Wiley.

H. Goldstein (1950), Classical Mechanics, Addison-Wesley.

W. Hamilton (1833), 'On a General Method of Expressing the Paths of Light, and of 
the Planets, by the Coefficients of a Characteristic Function', Dublin University Review, October 1833, 795-826.

W. Hamilton (1834), 'On the Application to Dynamics of a General Mathematical Method previously Applied to Optics', British Association Report, 1834, 513-518.

M. Herzberger (1958), Modern Geometrical Optics, Interscience Publishers.

P. Holland (1993), The Quantum Theory of Motion, Cambridge University Press.

P. Holland (2001), 'Hamiltonian Theory of Wave and Particle in Quantum Mechanics Part I', Nuovo Cimento 116B 1043-1069.

P. Holland (2001a),'Hamiltonian Theory of Wave and Particle in Quantum Mechanics Part II', Nuovo Cimento 116B 1143-1172.

F. John (1971), Partial Differential Equations, Springer Verlag.

M. Kline (1970), Mathematical Thought from Ancient to Modern Times, Oxford University Press.

C. Lanczos (1986), The Variational Principles of Mechanics, Dover (4th edition).

R. Littlejohn (1992), 'The Van Vleck Formula, Maslov Theory and Phase Space Geometry', Journal of Statistical Physics 68, 7-50.

J. Logan (1994), An Introduction to Non-linear Partial Differential Equations, John Wiley.

A. Messiah (1966), Quantum Mechanics, vol. I, North Holland: John Wiley.

H. Rund (1966), The Hamilton-Jacobi Theory in the Calculus of Variations, Van Nostrand.

I. Stakgold (1967), Boundary Value Problems of Mathematical Physics, Macmillan.

J. Synge (1954), Geometric Mechanics and deBroglie Waves, Cambridge University Press.

M. Taylor (1996), Partial Differential Equations, volume 1, Springer Verlag.

A. Webster (1950), Partial Differential Equations of Mathematical Physics (ed. S.Plimpton),

Hafner.

E. Whittaker (1952), A History of the Theories of Aether and Electricity, volume 1, Nelson.

E. Whittaker (1959), A Treatise on the Analytical Dynamics of Particles and Rigid Bodies, Cambridge University Press (4th edition). 